\title{
Sur Monostoma petasatum Deslongchamps 1824 et son cycle évolutif à deux hôtes
}

\author{
Par Robert-Ph. DOLLFUS
}

Eudes Deslongchamps (1824, p. 551, $\mathrm{n}^{\circ} 2$ ) a sommairement décrit, sans figure, un Monostome qu'il avait trouvé adulte dans les caeca d'Haematopus ostralegus L. (Charadriidae), à Caen (Calvados). Il le nomma Monostoma petasatum (*).

F. Dujardin (1845, p. 350-351) a reproduit la description originale de Deslongchamps mais l'a mal interprétée et a supposé que l'orifice antérieur, mentionné par Deslongchamps, était une ventouse ventrale et «qu'un orifice buccal se trouvait à l'extrémité du bord antérieur »; il en a déduit qu'il s'agissait probablement d'un Holostome voisin de l'Holostomum denticulatum (Rudo!phi 1819).

Quelques exemplaires d'un Monostome, que nous admettons être celui de Deslongchamps, ont été trouvés à Roscoff (Finistère) par Alfred Villot (1878, p. 18-20, pl. V, fig. 1), dans l'intestin d'un Strepsilas interpres (L.) (1). La figure donnée par Villot a été reproduite par Max Braun (1893, pl. XXVI, fig. 1). C'est la description et la figure données par Villot qui ont permis l'identification de l'espèce par les helminthologistes qui l'ont retrouvée et signalée: W. Nicoll (1907, p. 248) dans les caeca de Totanus calidris (L.), à Saint-Andrews (Ecosse), et Marie V. Lebour (1909, p. 3) dans les caeca de Strepsilas interpres (L.), à Beadnell (Northumberland) (2). Ni Nicoll, ni Lebour n'ont proposé une nouvelle attribution générique ; ils ont seulement employé l'appellation Monostomum petasatum Deslongchamps et, dans sa « Reference list of the Trematode parasites of British Birds », W. Nicoll $(1923$, p. 171, 190) a gardé le même nom, le plaçant parmi les « unclassified Monostomes ». Les ouvrages généraux sur les Trématodes, te's que ceux de Ben Dawes (1946) et de S. Yamaguti (1958), ne mentionnent même pas l'existence de ce parasite.

(1) Dans une note préliminaire, Villot (1875, p. 477) avait seulement relaté qu'il avait trouvé un «Monostomum à tête ailée et large ventouse dans l'intestin d'un Strepsilas interpres (L.).

(2) Il est vraisemblable que d'autres helminthologistes ont retrouvé ce parasite, mais ne l'ont pas signalé ou identifié. Par exemple, le Monostomum sp. trouvé par Einar Lönnberg (1890) chez Haematopus ostralegus L. à Bergen Glaesvaer (côte ouest de Norvège) était, peut-être, l'espèce de Deslongchamps; peut-être aussi le Monostomum ignotum W. Nicoll (1906, p. 514), du même hôte, à Saint-Andrews (Ecosse).

* Dans une autre publication, E. Deslongchamps (1827, p. 101-102) a relaté avoir trouvé un Monostoma à tête subtrigone dans les cæca d'Haematopus ostralegus L.; il en a donné une description qui n'est pas meilleure que celle de 1824 et il a choisi un autre nom spécifique, disant: « Nous lui avons donné l'épithète spécifique Trigonocephalum.» Ce nom est heureusement resté ignoré des helminthologistes. 
A vrai dire, la description et la figure données par Villot n'étaient pas suffisamment détaillées pour permettre soit l'attribution à un genre déjà existant, soit la création d'un nouveau genre. Les réviseurs de l'ancien genre « Monostoma » (Fr. Sav. Monticelli 1892, G. Brandes 1892) ont mentionné l'espèce de Deslongchamps sans se prononcer à son sujet; toutefois, quelques suppositions sur la position systématique ont été hasardées dubitativement: A. Looss (1902, p. 610) a fait remarquer que la forme de la tête, sans plus, rappelait celle des Pronocéphalidés; mais le rattachement à cette famille n'était pas possible, malgré la forme de la tête rappelant celle d'espèces de la famille Pronocephalidae A. Looss 1902 (par exemple des genres Pleurogonius A. Looss 1901 et Adenogaster A. Looss 1901), parce que, dans cette famille, entre autres caractères différentiels, l'ovaire est toujours en avant des testicules et un peu latéral, le vitelloducte transverse est en arrière de l'ovaire, le pore génital est plus ou moins latéral.

Willy Kossack (1911, p. 553) a rapproché l'espèce de Deslongchamps des Notocotylidae Max Lühe (1909, p. 31), mais les différences, ainsi que nous le verrons plus loin, sont, malgré des analogies évidentes, trop importantes pour permettre l'attribution à l'unique sous-famille Notocotylinae W. Kossack (1911, p. 22 ; 1911, p. 554-555) de cette famille.

Une meilleure connaissance anatomique de ce Monostome me paraissant indispensable pour préciser ses affinités, j'ai estimé nécessaire de revoir le matériel anciennement récolté par Alfred Villot à Roscoff, en septembre 1874.

Une partie de ce matériel existant encore au Laboratoire de Zoologie de l'Université de Grenoble, j'en ai demandé communication au Professeur André Dorier et celui-ci a eu l'amabilité de me confier - ce dont je le remercie vivement - les préparations, heureusement conservées, de la collection Villot. Ce matériel comprend quatre spécimens : deux d'entre eux ont été montés en milieu liquide et sont devenus inutilisables, les deux autres ont été montés dans du baume du Canada; toute coloration a disparu et ils sont devenus si transparents que quelques détails de leur organisation ne sont plus perceptibles; néanmoins, ils permettent de donner une description et des figures meilleures que celles publiées par Villot.

\section{Description}

Corps plat, allongé, plus de trois fois à trois fois et demie plus long que large (mesurant $4,5 \times 0,75 \mathrm{~mm}$ d'après Deslongchamps, $4 \times 1 \mathrm{~mm}$ d'après Villot).

Bords latéraux non parallèles mais un peu festonnés et s'élargissant lentement en direction postérieure. Extrémité antérieure pourvue, de chaque côté, d'une sorte d'aileron, dont le bord s'étend transversalement dans toute la largeur de la tête, à la face ventrale, « ce qui donne à la tête du ver, dit Villot, lorsqu'on la regarde de face, une vague ressemblance avec un chapeau », d'où le nom spécifique donné par Deslongchamps. L'extrémité postérieure est arrondie et un peu plus large que l'antérieure.

La cuticule n'est pas spinulée. Je n'ai pas vu de glandes cutanées comme il y en a chez Notocotylus. 
La ventouse orale est grande (diamètre: $0,32 \mathrm{~mm}$ d'après Villot) et profonde, elle occupe toute la hauteur de la tête, de l'apex au rebord du chapeau. L'œsophage qui lui fait suite est moins long que le diamètre de la ventouse; il n'y a pas de pharynx. Les caeca intestinaux restent distants des bords latéraux du corps; ils sont à peu près rectilignes, sauf tout à fait postérieurement, où ils se rapprochent quelque peu; ils se terminent sans atteindre l'extrémité postérieure du corps.

Il y a deux testicules situés dans le dernier cinquième de la longueur du corps ; ils sont plus longs que larges et à bords irrégulièrement sublobés; ils sont en dehors des caeca intestinaux et dépassent l'extrémité postérieure de ceux-ci.

La poche du cirre, mal visible chez mes individus, paraît courte ; elle aboutit au pore génital, situé au milieu de la largeur du corps, à la fin du premier sixième ou septième de la longueur de celui-ci. Je n'ai pas pu voir clairement une vésicule séminale interne dans la partie proximale de la poche du cirre.

L'ovaire est situé entre les deux testicules, au même niveau; il en est séparé par les caeca intestinaux; il est plus petit que les testicules et son contour est irrégulier.

Les vitellogènes sont situés de part et d'autre des caeca intestinaux et formés de follicules de forme irrégulière, ils s'étendent sur environ un quart à environ un sixième de la longueur du corps, immédiatement en avant des testicules. Le vitelloducte transverse passe contre le bord antérieur de l'ovaire. Le réservoir vitellin et la glande de Mehlis sont médians.

L'utérus a un trajet ascendant en spirale, décrivant des sinuosités très serrées, transversales, occupant la largeur de l'espace intercaecal, sauf distalement, où elles s'étendent sur une moindre largeur, avant de joindre la poche du cirre jusqu'au pore génital, en arrière de la bifurcation intestinale.

Les œufs remplissent l'utérus, ils sont extrêmement nombreux, operculés, très petits, mesurant de 20 à $22 \mu$ de long sur 12 à $13 \mu$ de large. La coque est très mince (environ $1 \mu$ ). Je ne suis pas certain de l'absence de filaments polaires, mais je n'en ai pas vu.

En ce qui concerne l'appareil excréteur, je ne l'ai observé qu'en partie ; il y a, en arrière de l'ovaire, une vessie terminale allongée longitudinalement, pourvue ou non d'une ou deux paires de très courts diverticules latéraux; deux gros canaux y aboutissent symétriquement; ils passent d'abord entre les caeca et les testicules, puis en dehors des vitellogènes; ils se maintiennent dans l'espace entre les bords latéraux du corps et les caeca, jusqu'au niveau de l'œsophage. Le pore excréteur est ventral, un peu en avant de l'extrémité du corps. 

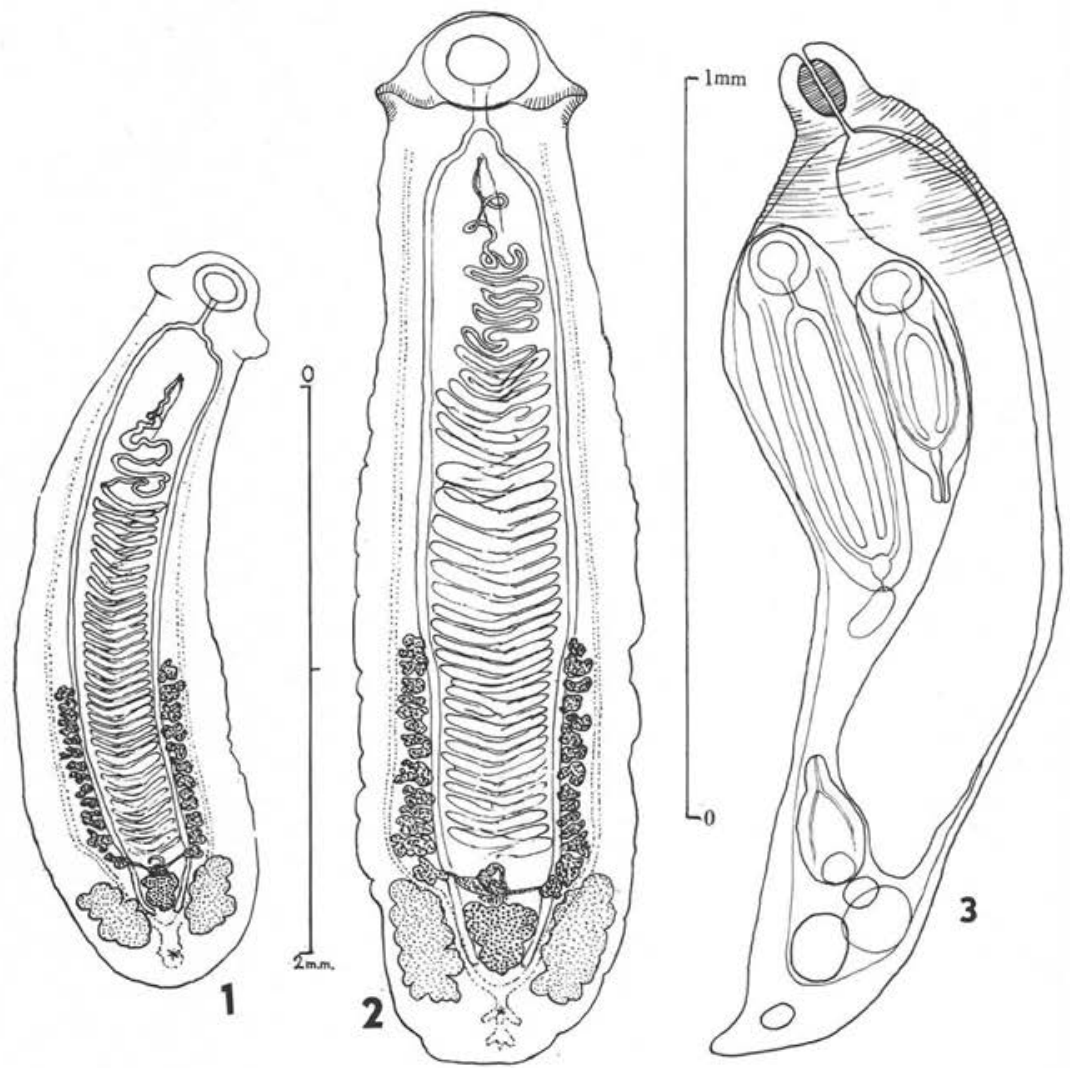

FIG. 1 et 2. - Notocotyloides petasatum (E. Deslongchamps 1824), adultes, récoltés à Roscoff (Finistère) chez Strepsilas interpres (L., 1758) par Alfred Villot (sept. 1874)

FIG. 3. - Rédie (longueur : 1,35, largeur : 0,3 au niveau de la mi-longueur, pharynx : $0,065 \times 0,045 \mathrm{~mm})$ de la glande génitale de Littorina obtusata (L. 1758) à Saint-Vaastla-Hougue (Manche), ipse legi, sept. 1912. Chez une cercaire en formation, on voit que le canal excréteur terminal passe dans l'appendice caudal

\section{Stades larvaires}

Dans le foie et la glande génitale de Littorina obtusata (L.), à Saint-Vaast-laHougue (Manche) (3), j'ai trouvé des rédies produisant des cercaires et, dans la cavité générale, j'ai trouvé la métacercaire correspondante. Une de ces littorines hébergeait

(3) A Saint-Vaast-la-Hougue, entre l'île Tahitou et le rivage, Littorina obtusata (L.) a son habitat optimum dans la zone de Fucus platycarpus Thuret, mais on en trouve depuis le niveau de Pelvetia canaliculata L. jusqu'à celui des plus hauts Fucus serratus L. J'ai surtout examiné des spécimens récoltés sur les Fucus vesiculosus L., qui sont à découvert presque à toutes les marées basses, même en morte eau. Cette station est très fréquentée par des Charadriiformes, tels que Strepsilas interpres (L.) et Haematopus ostralegus L., dont une partie de la nourriture consiste en Littorines. 

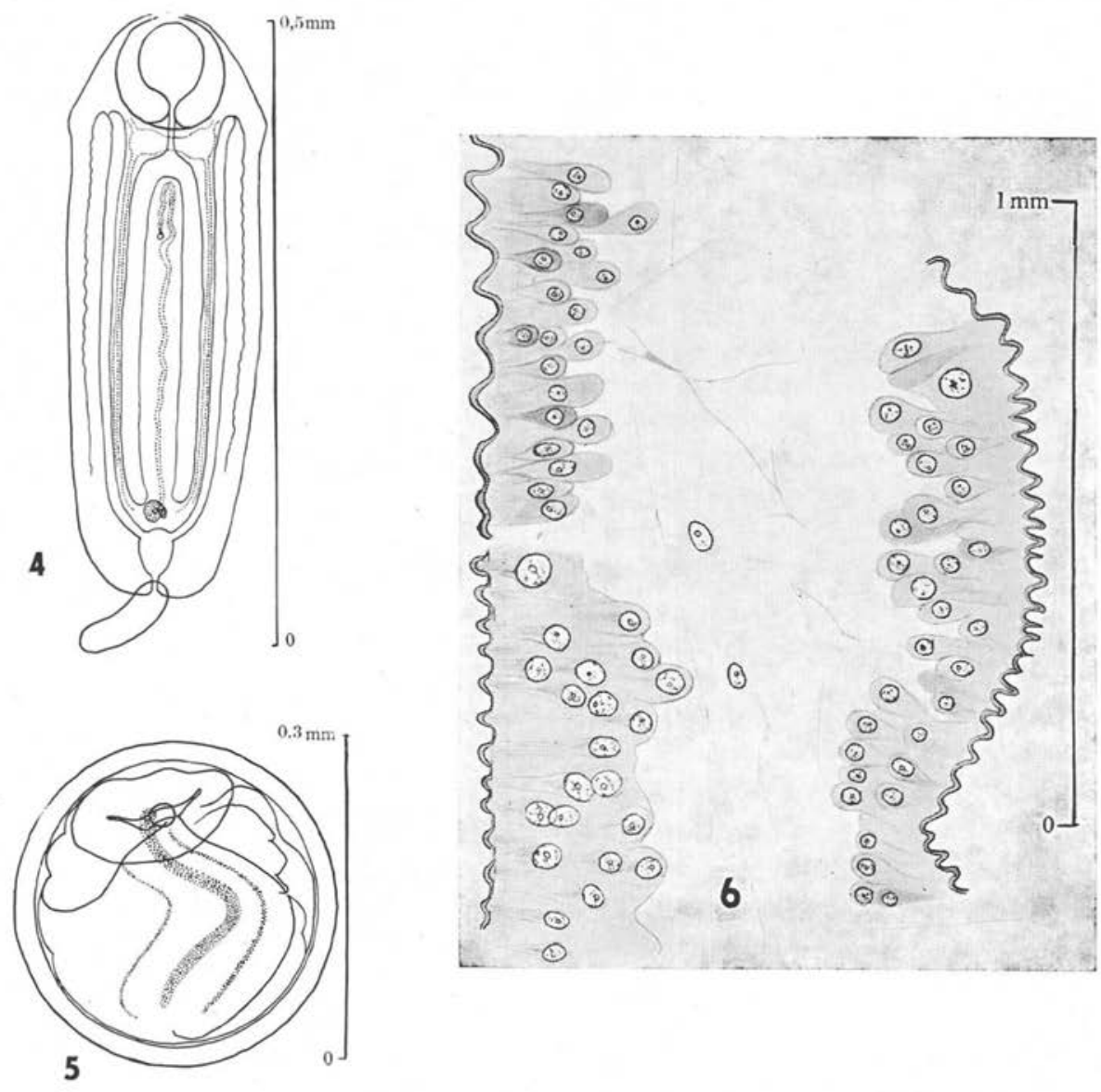

FIG. 4. - Cercaire âgée, d'après un dessin in vivo complété par un dessin du même individu après fixation. L'ébauche utérine se présente sous forme de deux files longitudinales de noyaux

Fig. 5. - Métacercaire dans son kyste

Fig. 6. - Aspect, sur des coupes obliques, de la paroi d'une Rédie fortement contractée. Chaque feston de la ligne extérieure correspond à la rencontre de deux fibres musculaires à directions perpendiculaires qui se croisent

au moins une centaine de cercaires et un grand nombre de métacercaires enkystées. Même chez les littorines dont la glande génitale était complètement envahie par des rédies, il n'y avait pas castration parasitaire complète.

Les rédies sont plus ou moins subcylindriformes à claviformes; leur extrémité antérieure est rétrécie au niveau du pharynx, ensuite le corps s'élargit, puis se rétrécit lentement pour se terminer en pointe mousse. Les plus grandes rédies que j'ai mesurées n'atteignaient pas une longueur de $1,5 \mathrm{~mm}$, la plupart étaient longues d'environ $1 \mathrm{~mm}$, larges de 1 tiers de $\mathrm{mm}$; il y en avait de beaucoup plus petites, très jeunes. 
A l'apex, l'orifice buccal conduit presque immédiatement au pharynx, ellipsoïdal, auquel fait suite un œsophage plus court que le pharynx, aboutissant à la poche gastrique, très allongée, qui s'étend généralement assez loin (jusqu'aux quatre cinquièmes environ de la longueur).

A mesure que les cercaires se développent dans la cavité générale, la poche gastrique subit une compression qui diminue son volume. Je n'ai observé aucune granulation, aucune particule solide dans la poche gastrique.

Le nombre des cercaires complètement développées dans la cavité générale varie de trois à six. C'est seulement dans le cul-de-sac postérieur de la rédie que prennent naissance les cercaires. Les embryons de cercaires grandissent pendant quelque temps, inclus dans le parenchyme du cul-de-sac de la cavité avant d'être libérés dans la cavité où ils atteindront leur complet développement. Je n'ai pas observé de pore d'accouchement.

La paroi de la rédie est très contractile. La contraction produit des renflements claviformes contenant chacun un noyau, qui font saillie dans la cavité générale de la rédie et qui correspondent chacun à un faisceau musculaire (4). Ce sont des éléments somatiques qui ne prennent pas part à la formation des embryons de cercaires.

La cercaire est un peu aplatie dorso-ventralement; elle est à peu près trois fois plus longue que large.

Dimensions de la cercaire après fixation (en $\mathrm{mm}$ ) :

\begin{tabular}{|c|c|c|c|}
\hline Longueur du corps & 0,485 & 0,480 & 0,630 \\
\hline Largeur du corps $\ldots \ldots \ldots$ & 0,140 & 0,160 & 0,200 \\
\hline Ventouse orale, diamètre $\ldots \ldots \ldots \ldots \ldots \ldots$ & 0,07 & 0,10 & 0,13 \\
\hline Esophage, longueur ........... & 0,02 & 0,01 & 0,03 \\
\hline Queue, longueur ..... & 0,07 & 0,08 & 一 \\
\hline Queue, largeur $\ldots \ldots \ldots \ldots \ldots \ldots \ldots$ & 0,04 & 0,03 & - \\
\hline
\end{tabular}

Les deux extrémités sont arrondies. Les bords latéraux sont subparallèles, la largeur diminue légèrement en direction postérieure. La partie antérieure du corps est en forme de triangle curviligne, les angles latéraux montrent un léger renflement. La cuticule est lisse. L'extrémité postérieure porte un court appendice caudal ovale, un peu aplati, atteignant approximativement un septième de la longueur du corps. Il est peu mobile et facilement caduque ; il ne semble pas avoir un rôle natatoire. Chez la cercaire incomplètement formée, la queue est traversée par le canal évacuateur de la vessie.

Le parenchyme du corps étant très opaque, l'observation sur le vivant des cercaires à maturité ne permet pas de voir les organes, on remarque seulement trois bandes sombres, longitudinales, parallèles, dont les deux latérales correspondent aux caeca intestinaux et la médiane à l'ébauche de l'utérus, ainsi que le montrent les préparations d'individus rendus transparents.

(4) Comparer, par exemple, avec la structure du sporocyste d'un Tergestia sp., décrit par W. A. Haswell (1903, p. 499, pl. XIX, fig. 8), montrant un semblable aspect. Haswell a estimé qu'il s'agissait d'un « germinal épithélium »; mon opinion est qu'il s'agissait, comme dans le cas présent, d'éléments somatiques. 
La ventouse orale, très grande, à ouverture antéro-ventrale, occupe toute la hauteur du triangle céphalique. L'œsophage est très court et les deux caeca s'étendent parallèlement jusqu'aux $7 / 8$ de la longueur du corps. En dehors de chaque caecum se trouve un gros canal excréteur, s'étendant parallèlement aux bords latéraux du corps jusqu'au niveau du bord postérieur de la ventouse. Ces deux gros canaux s'ouvrent séparément dans la vessie, qui est relativement petite, en forme de ballon, dans environ le dernier huitième de la longueur du corps et s'ouvre à l'extrémité postérieure, ventralement au point d'attache de l'appendice caudal. Chez les très jeunes cercaires, avant que le parenchyme ne soit devenu opaque, on voit, sur le vivant, un courant ciliaire dans les gros canaux. L'extrémité de ceux-ci reçoit un canal plus fin à trajet postéroantérieur, qui s'étend parallèlement aux bords latéraux du corps, en dehors des gros canaux.

Le système nerveux central est formé d'une bandelette transversale assez large, renflée à ses deux extrémités, située entre la ventouse orale et la bifurcation intestinale, dorsalement à l'œsophage.

Après coloration, quelques ébauches génitales sont observables ; l'utérus est représenté par une double file de noyaux sur la ligne médiane, débutant un peu en avant du bord antérieur de la vessie et se terminant un peu en arrière de la bifurcation intestinale. Un groupe de noyaux, médian entre la terminaison des caeca et la vessie, représente l'ébauche de l'ovaire, de la glande de Mehlis et du réservoir vitellin. L'ébauche de la poche du cirre est visible sur la ligne médiane, vers la limite postérieure du premier tiers de la longueur du corps. Les ébauches testiculaires et celles des vitellogènes ne sont pas distinctes.

Les cercaires mûres quittent les rédies mais ne gagnent pas le milieu extérieur: elles perdent leur queue et se répandent dans la cavité générale de la Littorine. Elles s'y enkystent. Le kyste est sphérique, à paroi épaisse ; son diamètre moyen est d'environ $0,3 \mathrm{~mm}$. Par transparence, on voit la métacercaire repliée et enroulée sur ellemême. Les kystes métacercariens ont toujours été observés très nombreux dans les Littorines parasitées par des rédies.

La métacercaire présente déjà les caractéristiques principales de l'adulte immature ; l'utérus n'a pas encore sa forme et son étendue définitives.

Les Charadriiformes, hôtes définitifs, s'infestent par ingestion de Littorines contenant des métacercaires.

C'est donc un cycle évolutif à deux hôtes (5).

\section{Discussion. Position systématique}

L'anatomie de l'adulte diffère à peine de celle des Notocotylidae du groupe Notocotylus-Catatropis. Les organes ont la même disposition, les mêmes rapports. Remar-

(5) Ma découverte du cycle évolutif de Monostoma petasatum Deslongchamps a été sommairement exposée dans ma notice: "Titres et travaux scientifiques» $(1941$, p. 39) et il en a été fait mention dans le «Rapport de la Commission pour le Prix Jules de Guerne », prix qui m'a été attribué en 1925 (Bull. Soc. zool. France, t. L, no 3, 15-VI-1925, p. 61). 
quons, toutefois, qu'il n'y a pas une très longue poche du cirre et que la forme de la tête est complètement différente.

La même forme de la tête existe dans le genre Parapronocephalum Belopolskaïa, dont seulement une espèce est connue : P. symmetricum Belopolskaïa 1952 (p. 141-143, fig. 6), qui a été trouvée dans les caeca intestinaux de Calidris [= Arquatella] maritima (Brünnich 1764) de la côte Mourmane par M. M. Belopolskaïa et d'Arenaria interpres (L.) de la mer Blanche par D. V. Naumov.

Disposant d'un abondant matériel, en très bon état, Belopolskaïa a donné une description détaillée de cette espèce. Elle l'a admise dans les Pronocephalinae A. Looss 1899, la raprochant plus particulièrement du genre Adenogaster A. Looss 1901, créé pour une seule espèce: A. serialis A. Looss 1901 (connue seulement à l'état adulte), de la première moitié du gros intestin d'un Thalassochelys corticata (Rondelet) à Alexandrie (Egypte).

Chez ce Trématode, la face ventrale présente - ce qui est exceptionnel chez les Pronocephalinae - des séries longitudinales de glandes cutanées (comme il y en a chez Notocotylus) d'où le nom spécifique donné par Looss.

Bien que la forme de la tête soit quelque peu voisine chez Adenogaster et Parapronocephalum et qu'il y ait des séries longitudinales de glandes cutanées dans les deux genres, il y a de grandes différences anatomiques qui les séparent: chez Adenogaster, les caeca digestifs sont pourvus de sacculations le long de leur bord interne, les testicules sont presque complètement extracaecaux, le vitelloducte transverse est en arrière de l'ovaire, qui est un peu déplacé vers la droite, le pore génital est déplacé vers la gauche, etc... C'est pourquoi Belopolskaïa, ne pouvant pas placer son espèce dans un genre de Pronocephalinae, a proposé un nouveau genre, qu'elle a placé dans cette sous-famille.

L'attribution aux Notocotylinae n'aurait pas été acceptable, non pas seulement parce que les testicules de Parapronocephalum sont intracaecaux, alors qu'ils sont extracaecaux chez les Notocotylinae, mais parce que la cercaire est d'un type complètement différent. Chez les Notocotylidae, la cercaire a une queue natatoire aussi longue ou plus longue que le corps et une paire d'yeux latéraux pigmentés ou, pour quelques espèces, un œil médian en plus de la paire latérale. Ces yeux persistent chez la métacercaire.

Belopolskaïa, sans avoir vu la cercaire de Parapronocephalum, savait que celle-ci ne ressemblait pas aux cercaires de Notocotylinae. En effet, Belopolskaïa, à défaut de cercaire, avait trouvé et identifié la métacercaire dans le foie de Littorina rudis Maton, de la côte Mourmane, et avait observé de très jeunes immatures, différant à peine de la métacercaire, dans le gosier de Calidris $[=$ Arquatella $]$ maritima (Brünnich 1764). Les métacercaires de la Littorine mesuraient $1,232 \times 0,296 \mathrm{~mm}$ et les immatures de Calidris 1,408 $\times 0,312 \mathrm{~mm}$ (voir Belopolskaïa 1952, p. 142, 175-176, tableau 22 des dimensions des métacercaires de la Littorine et des immatures du gosier de Calidris, fig. $11 a$ chez Calidris, fig. $b$ chez la Littorine).

Peu après, tous les stades larvaires furent trouvés par G. K. Chubrik (1954, p. $565-567$, fig. $1 a-b$ rédies, $1 v$ cercaire avec son appendice caudal, $1 g$ cercaire ayant 
perdu son appendice caudal, fig. $1 d$ métacercaire dans son kyste) chez des Littorina saxatilis Olivi (= rudis Maton) de la côte Mourmane et de la mer Blanche, dans le foie et la gonade. Chubrik remarqua qu'en été on trouvait les rédies et cercaires chez les Littorines, mais qu'en automne on ne trouvait plus que les métacercaires.

Si l'on compare les stades larvaires décrits et figurés par Chubrik avec ceux de Monostoma petasatum Desl., on constate qu'ils sont, à très peu près, semblables.

Malgré cette presque identité des formes larvaires et la même forme particulière de la tête, je ne crois pas que l'espèce de Deslongchamps soit congénérique de celle de Belopolskaïa. Nous avons vu que, chez celle-ci, les testicules sont intracaecaux et que trois séries longitudinales de glandes cutanées sont présentes à la face ventrale ; ce sont des caractères s'opposant à ceux de petasatum. Je propose donc, pour petasatum, un nouveau genre: Notocotyloides, et je le place dans une nouvelle famille: Parapronocephalidae, sous-famille Parapronocephalinae K. I. Skrjabin (1955, p. 210) (6), dans le sous-ordre Notocotylata K. I. Skrjabin \& R. Ed. Schulz 1933 (7), qui contient déjà cinq familles.

L'emplacement de cette nouvelle famille est schématisé ci-après :

- Parasites de Pécilothermes: Reptiles, rarement Poissons (chez les Poissons, seulement Barisomum Linton 1910, Himasomum Linton 1910).

Stade Cercaria inconnu.

Adulte avec expansions céphaliques (peu d'exceptions) ........ Pronocephalidae.

- Parasites d'Homéothermes: Oiseaux, rarement Mammifères.

Stade Cercaria avec longue queue et yeux pigmentés.

Adulte sans expansions céphaliques

Notocotylinae.

Stade Cercaria avec queue rudimentaire, pas d'yeux.

Adulte avec expansions céphaliques

Parapronocephalinae.

La diagnose de Notocotyloides n. gen., type petasatum Deslongchamps 1824 est : * Parapronocephalinae ayant les caractères de Parapronocephalum, sauf que les testicules sont extracaecaux et que les glandes cutanées sont absentes ».

(6) K. I. Skrjabin (1955, p. 201-210) a longuement discuté des affinités naturelles et de la position systématique de Parapronocephalum; il a conclu que l'existence d'un collier céphalique dans ce genre n'était pas un caractère assez important pour justifier une sous-famille Parapronocephalinae, séparée des Notocotylinae.

(7) $\mathrm{Ce}$ sous-ordre est l'équivalent de la superfamille Notocotylidae F. Poche 1926 (nom changé en Notocotylidea par E. W. Price, 1932, p. 54, note 4) = Pronocephaloidea J. M. Ruiz 1946. Actuellement, on n'admet plus dans les Pronocephalinae de parasites d'homéothermes et l'on conserve la fam. Rhabdiopoeidae F. Poche 1926 pour les parasites de Siréniens; cette famille comprend les genres Rhabdiopoeus S. I. Johnston 1913 et Taprobanella H. Cruz et V.S. V. Fernand 1954 ; ce dernier genre avait été considérée par ses auteurs comme appartenant aux Pronocephaliinae et ils en avaient donné une nouvelle définition permettant d'y inclure une espèce parasite d'homéotherme. 


\section{Résumé}

Monostoma petasatum Eudes Deslongchamps 1824 ayant été incomplètement décrit, n'avait pas reçu d'attribution générique. J'en donne une meilleure description, d'après des spécimens récoltés à Roscoff par A. Villot en septembre 1874, chez Strepsilas interpres (L.). Je décris, en outre, la rédie, la cercaire, la métacercaire, que j'ai trouvées à Saint-Vaast-la-Hougue (Manche) en septembre 1912 chez Littorina obtusata (L.). Le cycle n'a que deux hôtes.

Je propose le nouveau genre Notocotyloides, que je place avec Parapronocephalum M. Belopolskaïa dans la sous-famille Parapronocephalinae K. I. Skrjabin 1955, famille Parapronocephalidae nova, dans le sous-ordre Notocotylata K. I. Skrjabin \& R. Ed. Schulz 1933.

\section{Bibliographie}

BelopolsKaïa (M. M.), 1952. - Parasitofaune des oiseaux de mer nageurs. Utchenié Zapisky Leningradsk Gosudartsv. Univers., 1952, $\mathrm{n}^{\circ}$ 141. Série des Sciences biologiques, livraison 28, p. 127-180, fig. 1-13.

Braun (Maximilian), 1893. - Plathelminthes. I. Trématodes. Bronn's Klassen und Ordnungen des Thier Reichs, IV. Bd. Vermes. Abth. Ia, 1889-1893. p. 303-925 + I-v, fig. texte 1-36, pl. I-XXXIV.

Chubrik (G. K.), 1954. - Le cycle évolutif du Trématode Parapronocephalum symmetricum Belopolskaïa 1952. - Doklady Acad. Sciences, U.R.S.S., nouv. sér., t. XCVII, $\mathrm{n}^{\circ} 3$, 1954, p. 565-567, fig. 1 a-1 $\mathrm{g}$.

Deslongchamps (Eudes), 1824. - Monostome, Monostoma. Encyclopédie méthodique. HisNaturelle des Zoophytes ou animaux rayonnés, faisant suite à l'Histoire Naturelle des Vers de Bruguière, t. II, Paris 1824, p. 551-555.

-, 1827. - Monostome, Monostoma. Dictionnaire classique d'Histoire Naturelle, Paris. vol. XI, janv. 1827, p. 101-102.

Dujardin (Félix), 1845. - Histoire naturelle des Helminthes. Suites à Buffon, Roret, Paris, 1845 , XVI +654 p., Atlas, 15 p., pl. I-XII.

HASWELl (William, Aitcheson), 1903. - On two remarkable sporocysts occurring in Mytilus latus, on the coast of New Zealand. Proc. linn. Soc. New South Wales, $\mathrm{n}^{\circ} 108$, vol. XXVII, part 4 (29 oct. 1902), april 9, 1903, p. 497-515, pl. XIX, fig. 1-15 ; pl. XX, fig. 16-36.

Kossack (Willy), 1911. - Ueber Monostomiden. Inaug. Dissert. Albertus Univ. Königsberg, 1911, p. 1-32, fig. 1-4.

-, 1911. - Ueber Monostomiden. Zoolog. Jahrbücher, Abt. System., Bd XXXI, Heft 4, 31-10-1911, p. 491-590, pl. XIII-XV, fig. 1-26.

Lebour (Marie-V.), 1909. - Trematodes of the Northumberland coast. $\mathrm{N}^{\circ}$ III. A preliminary note on Echinostephilla virgula, a new Trematode in the Turnstone. Transact. Nat. Hist. Soc. Northumberland, Durham and Newcastle-upon-Tyne, New Series, vol. III, part. 3 , march 1909 , p. 3-8 (440-445), pl. XI, fig. 1-3. 
LoENNBerg (Einar), 1890. - Helminthologische Beobachtungen von der Westkuste Norwegens. Erster Teil. Cestoden. Bihang till. k. Svenska Vet. Akad. Handlingar, Stockholm, Bd XVI, Afd. IV, $\mathrm{N}^{\circ} 5,1890$, p. 1-47.

Looss (Arthur), 1901. - Ueber Trematoden aus Seeschildkröten der egyptischen Küsten (Notizen zur Helminthologie Egyptens 4). Centralbl. für Bakt. Parasitenkunde, I. Abt., Bd XXX, Heft 15, 30-X-1901, p. 555-569; Heft 16, 7-XI-1901, p. 618-625.

- 1902. - Ueber neue und bekannte Trematoden aus Seeschildkröten. Nebst Erörterungen zur Systematik und Nomenclatur. Zoologische Jahrb., Abt. System., Bd XVI, Heft 3-6, 24-XI-1902, p. 411-894, fig. texte A-B, pl. XXI-XXXII, fig. 1-181.

LüHe (Max)., 1909. - Parasitische Plattwürmer. I.: Trematodes. Die Süsswasserfauna Deutschlands, Heft 17, Jena 1909, p. 1-217, fig. 1-188.

Nicoll (William), 1906. - Some new and little-known trematodes. Ann. and Mag. Nat. Hist., ser. 7, $\mathrm{n}^{\circ}$ 102, vol. XVII, june 1906, p. 513-527, pl. XII-XIII, fig. 1-10.

-, 1907. - Observations on the trematodes parasites of British Birds. Ann. and Mag. Nat. Hist., ser. $7, \mathrm{n}^{\circ} 117$, vol. XX, sept. 1907, p. 245-271.

-, 1923. - A reference list of the Trematodes parasites of British birds. Parasitology, vol. XVI, $\mathrm{n}^{\circ}$ 2, june 1923, p. 151-202.

PrICE (Emmett W.), 1932. - The Trematode parasites of marine Mammls. Proc. U.S. Nat. Museum, $\mathrm{n}^{\circ}$ 2936, vol. LXXXI, art. 13, 1932, p. 1-68, pl. I-XII, fig. 1-53.

SKRJABIN (Konstantin Ivanovitch), 1955. - Trématodes des animaux et de l'homme. Bases de la Trématodologie. Edition de l'Académie des Sciences de l'U.R.S.S., Moscou, t. X, 1955, p. 1-653, fig. 1-175.

Villot (Alfred), 1875. - Sur les migrations et les métamorphoses des Trématodes endoparasites marins. Comptes rendus hebd. Acad. Sciences, Paris, t. LXXXI, n ${ }^{\circ} 11,13-$ IX-1875, p. 475-477.

—, 1878. - Organisation et développement de quelques espèces de Trématodes endoparasites marins. Ann. Sc. nat., Zoologie, $6^{\circ}$ sér., t. VIII, n 2, février 1878, p. 1-40, pl. V-X.

(Muséum National d'Histoire Naturelle, 57, rue Cuvier. F-75 - Paris, 5). 